\title{
Graminées du Nord Cameroun et leurs utilisations
}

\author{
par PIERRE MALZY \\ Maitre de Recherches des Laboratoires du Service de l'Agriculture \\ de la France d'Outre-Mer
}

Les graminées, tant par leur nombre que par leurs utilisations fourragères, revêtent pour les populations du Nord Cameroun une importance particulière.

Il nous a paru intéressant de récolter au cours de nos déplacements les principales graminées, de noter leurs utilisations et leur valeur en tant qu'aliment du bétail.

Nous avons voulu réaliser, non pas une simple énumération, mais un travail pratique, susceptible d'être utilisé par ceux, privés ou administratifs, qui, par leur métier, s'intéressent à la couverture végétale du sol.

Ce travail est limité à la région comprise entre la base de la falaise de N'Gaoundéré et le lac Tchad, soit dans la partie connue habituellement sous le nom de plaine du Tchad.

Il nous est agréable de remercier ici M. R. Portères, professeur au Muséum National d'Histoire Naturelle et $M$. Jacques-Félix, directeur de laboratoire a la Section Technique d'Agriculture Tropicale, qui ont bien voulu effectuer la détermination de la plus grande partie des graminées recoltees.

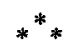

1. Acroceras amplectens Stapf. - (Burgu Danehiel) (1). La plante est consommée en herbe et en grains par les chevaux, bœufs, moutons, chèvres (Garoua 592) (2).

2. Andropogon gayanus Kunth var. genuinus Hackel. - (Seno = Senore.) La paille est utilisée pour la fabrication des « seccos » de clôture, pour la couverture des cases. A l'état jeune seulement la plante est pâturée par les chevaux, bœufs, moutons, chèvres (Garoua 503).

(1) Les noms entre parenthèses sont en "Fulfulde" ou Peul langue véhiculaire du Nord Cameroun. Ces noms sont donnés dans les deux dialectes: Funangere pour Maroua et Irnagere pour Garoua.

(2) Le nom et le numéro entre parenthèses se rapportent à l'herbier existant au Cenire de Garoua du Secteur Nord de Défense des Cultures.
3. Andropogon gayanus Kunth. - (Hore pul debbo [Garoua] = Dake pure [Garoua] $=$ Rubo [Garoua]). La paille est utilisée pour la confection de seccos de clôlure, pour la couverture des cases. La plante est consommée en herbe par les chevaux, bceufs, moutons, chèvres. En grains, elle est consommée par les bovins seulement (Garoua 509).

4. Andropogon pseudapricus Stapf. - (Djigaldji IGaroua] = Dioldoho [Maroua] = Tielbi [Garoua]). Ia paille est. utilisée pour la couverture des habitations. La plante est consommée, quand elle est jeune, par les chevaux, bovins, ovins, caprins ( $\mathrm{Ga}$ roua 502 )

5. Andropogon schirensis Hochst. - (Buhirdiho [Garoua] = Gati [Banyo]). La paille est utilisée pour la couverture des cases, pour la confection de seccos de cloture, pour la confection de balais. La plante est pâturée, en herbe seulement, par les bovins, ovins, caprins. Elle n'est pas consommée par les chevaux (Caroua 512).

6. Andropogon non déterminé. - (Mubarawal yolde [Garoua] $=$ Garlabal yolde [Maroua]). Graminée des terrains sablonneux, consommée en herbe et en grains par les chevaux, bceufs, moutons, chèvres (Garoua 599).

7. Aristida adscensionis Lin. - (Wytio dire « queue de rat palmiste " = Tielbi muratako tutako $=$ Datyere ngala). La plante n'est consommée par le bétail qu'en cas de pénurie d'autres fourrages (Garoua 197-570).

8. Aristida hordacea Kunth. - (Wytio dire bafere ([Garoua] $=$ Wytio dioldu [Maroua] $=$ Lamulamugel [Garoua]). Ia plante est consommée en herbe par les chevaux, bœufs, moutons, chevres. Les bovins, seuls, le consomment à l'état sec (Garoua 237, 469. 475),

9. Aristida kerstingii Pilger. - (Buhirdiho [Garoua] $=$ Tielbi danedyi [Maroua] $=$ Waloyi). La paille est utilisée pour la couverture des habitations, la confection de seccos de clôture, de balais (bu- 
hirdi). La plante est consommée par le bétail seulement en cas de disette d'autres fourrages (Garoua 177, 484).

10. Aristida longiflora Schum. et Thonn. - (Tielbi yolde [Garoua] = Tielbi danebi yolde [Maroua]). Graminée des terrains siliceux. La plante est consommée, en herbe seulement, par les chevaux, bceufs, moutons, chèvres (Garoua 199, 569).

11. Beckeropsis uniseta K. Schum, - (Mubarawal [Garoua] = Bulungel [Maroua] = Loklogal [Garoua] = Logoho [Garoua]). La paille est utilisée pour la couverture des cases. La plante est consommée en herbe et en grains, par les chevaux, bovins, ovins, caprins (Garoua 288, 498).

12. Brachiaria distichophylla Stapf. - (Furodo mayo). Graminćc dos bords des cours d'eau, consommée en herbe et en grains par les chevaux, bœufs, moutons, chèvres (Garoua 628.)

13. Brachiaria fulva Stapf. - (Sehuko aloyi [Garoua $]=$ Hieliyaho $[$ Maroua $]=$ Buhirdi [Garoua] $=$ Tiotihi tinikoy sadoyi [Garoua]). Graminée des terres humides. La paille est utilisée pour faire des chapeaux, des vans, des balais. La plante est consommée à l'état jeune et en grains par les bovins, ovins, caprins. Les chevaux ne la consomment qu'en herbe (Garoua 166, 497).

14. Brachiaria kotschyana Stapf. - (Sahaho kondorle $[$ Garoua $]=$ Hudo yolde $[$ Maroua $]=$ Wodeho tabedye). Graminée des terrains sablonneux, non consommée par le bétail (Garoua 253).

15. Brachiaria lata Hubb. - (Badeho diolde). Graminée fréquente sur les termitières. La plante est consommée, en herbe et en grains par les chevaux, bceufs, moutons, chèvres (Garoua 211, 513.)

16. Brachiaria mutica Stapf. - (Hebereho [Garoua] $=$ kewalwol [Garoua]). La plante est consommée en herbe et en grains, par les chevaux, boeufs, moutons, chèvres (Garoua 508).

17. Cenchrus biflorus Roxb. - « Cram-cram》(Kelbe [Garoua] $=$ kebbe hodedye $=$ Pegge wahi- . nabe [Maroua]). Graminée à graines munies de crochets, très envahissante. Elle est pâturée, en herbe, par les chevaux, bueufs, moulons, chèvres, Les terres où croit le « cram-cram» sont de bonne qualité pour la culture du petit mil et de l'arachide (Garoua 214, 621).

18. Chloris pilosa Schum et Th. - (Damaliliel yolde $\Rightarrow$ Damaliliel mayo). Graminée des terrains sableux proches des cours d'eau. La plante est consommée en herbe et en grains, par les chevaux, bceufs, moutons, chèvres (Garoua 181, 557).

19. Chloris pycnothrix Trin - (Damaliliel daneyel $=$ Damaliliel yolde). La plante est consommée, en herbe et en grains, par les chevaux, bœufs, moutons, chèvres (Garoua 293).
20. Chloris robusta Stapf. - (Sanganare = Lirontongel mayo [Maroua] = Tanganayel hiladi [Garoua]). Graminée des bords de cours d'eau. La paille est utilisée pour la confection de seccos de clôture, pour la couverture des cases (coussinets). La plante est pâturée, en herbe seulement, par les chevaux, bœufs, moutons, chèvres (Garoua 307, 535).

21. Ctenium canescens Benth. = (Butaliho walowol). Plante des terrains humides. La plante est consommée, en herbe et en grains, par les bovins seulement (Garoua 453).

22. Ctenium newtonii Hackel. - (Butaliho yolde). La plante qui pousse en terrains sablonneux n'est pas pâturée par les chevaux. Elle est consommée, en herbe et en grains, par les bœufs, moutons, chèvres (Garoul 175).

23. Cymbopogon citratus Stapf. - (Hudo saye $=$ Hudo ti). C'est la " citronelle ", bien connue des Européens : tisanes, bordures d'allees. Selon les croyances locales, l'infusion des feuilles calme la toux et soigne les maux de ventre. Les rhizomes sont utilisés comme brosses à dents, ils calment'les maux de dents. La plante n'est pas consommée par le bétail.

24. Cymbopogon giganteus Chiov. - (Wadjalo). Graminée odorante, connue en A.O.F. comme curatif de la fievre jaune, par frictions (Bambara : Bing fala $=$ Tiekala). La plante n'est pas consommée par le bétail. Non attaquée par les termites, la plante est utilisée pour la confection de seccos de clôture, pour la couverture des cases (coussinets). Le mélange suivant, en fumigation sur les braises, a la réputation de chasser les vers intestinaux : racines et feuilles de Cymbopogon giganteus, tiges d'Euphorbia kamerunica, chapeau de termitière champignon (Garoua 169).

25. Cynodon dactylon Pers. - (Nierhiello $=\mathrm{He}-$ dello mayo). « Chiendent pied de poule. » Les racines traçantes permettent la fixation des sols sablonneux, la constitution de pelouses. Selon les croyances locales, la décoction a une action calmante sur les jeunes enfants. L'a plante est pâturée, en herbe et en grains par les chevaux, bceufs, moulons, chèvres (Garoua 66, 207, 626).

26. Dactyloctenium aegyptium Beauv. - (Bagamri [Garoua] = Falande [Maroua]). Les graines, en cas de disette, servent à l'alimentation humaine, après réduction en farine. La plante est 'consommée, en herbe et en grains, par les chevaux, boufs, moutons, chèvres (Garoua 210).

27. Digitaria gayana Stapf. ex A. Chev. - (Hudo tabedye ranuhahore $=$ Kolitatiho [Maroua]). La plante est consommée en herbe et en grains, par les chevaux, boufs, moutons, chèvres (Garoua 567).

28. Digitaria lecardii Stapf. - (Hudo kossedye 
G $[$ aroua $]=$ Sewandande $[$ Maroua $]=$ Djeliyaho furda hore [Maroua] $==$ Saraho yolde [Garoua]). La plante est consommée, en herbe et en grains, par les chevaux, bœufs, moutons, chèvres (Garoua 182, 194).

29. Digitaria longiflora Pers. - (Walwalde mayo). Graminée des bords de cours d'eau. La plante est consommée en herbe et en grains, par les chevaux, boeufs, moutons, chèvres (Garoua 625).

30. Digitaria velutina Beauv. = Digitaria acuminatissima Stapf. - (Djeliyaho walowol $=$ Djeliyaho tabedye [Maroua]) = Sewadande [Garoua]). Graminée des terrains marécageux. La plante est consommée, en herbe et en grains, par les chevaux. bœufs, moutons, chèvres (Garoua 208)

31. Dinebra retroflexa Panzer. - (Lamulamugelho). Graminée consommée, en herbe et en grains, par les chevaux, boeufs, moutons, chèvres (Garoua $171,404,511)$.

32. Echinochloa colona Link, - (Paguri mayo = Paguri belli $=$ Paguri rabalde). Graminée des terres humides. Les graines, sous forme de farine, sont utilisées pour l'alimentation humaine, en cas de disette de céréales vivrières. La plante est consommée en herbe ef en grains, par les chevaux, houfs, moutons, chèvres (Garoua 217).

33. Echinochloa pyramidalis Hitch et Ch. - (Kaliari sadoyi $=$ Tagol $=$ Tagol bafere). Graminée poussant dans les terrains argileux. Il en existe une variété à épis violets. En cas de disette, les graines, sous forme de farine, sont utilisées pour l'alimentation humaine. La plante est pâturée, en herbe et en graines, par les chevaux, bœufs, moutons, chèvres (Garoua 236, 481, 483, 581).

34. Echinochloa stagnina P. Beauv. - (Burgu [Garoua] $=$ Gambarawal [Maroua]). Graminée existant en peuplement sur les terres périodiquement inondées (borgoutières) et constituant aux basses eaux d'excellents pâturages pour le bétail. Les tiges, de saveur sucrée, sont consommées crues (Garoua 333).

35. Echinochloa non déterminé. - (Burgu pabi [Garoua]). Graminée des terres inondées périodiquement, comme Echinochloa stagnina. Paturage apprécié pour tout le bétail (Garoua 69).

36. Echinochloa non déterminé. - (Pagamri bodehi walowol). Graminée des zones marécageuses. Les graines sont utilisées pour l'alimentation humaine, en cas de disette de céréales vivrières. La plante est consommée en herbe et en grains, par les chevaux, bœufs, moutons, chèvres (Garoua 640).

37. Eleusine coracana Gaertn. - (Tiargari = dudu [Maroua] $=$ en Fali : Titendari $=$ Tandari). Cette graminée, cultivée, est utilisée comme le mil dans l'alimentation humaine. Les grains sont donnés aux chevaux. Les Kirdis en font une boisson fermentée.
Les grains sont quelquefois consommés crus. La bouillie faite avec la farine sert aussi à soigner la diarrhée. Avec les tiges, on confectionne des vans. (Garoua 187, 582).

38. Eleusine indica Gaertn. - (Sargalde). La plante est consommée en herbe et en grains, par les chevaux, bœeufs, moutons, chèvres, mais'elle a la réputation de provoquer, chez les chevaux, des maux de dents. La tige est utilisée pour la confection de vans (Garoua 170, 495).

39. Elionurus elegans Kunth. - (Wodeho $=$ Hudo waloyi). Consommée en herbe et en grains, par les bovins, ovins, caprins, elle n'est pas consommée par les chevaux (Garoua 180).

40. Elytrophonus spicatus A. Camus. - (Hudo waloyi [Garoua] - Makorkoro [Maroua] = Butagelho [Maroua]). Localement sans utilisations, la plante n'est pas consommée par le bétail (Garoua 472).

41. Eragrostis aspera Nees. - (Hudo diahole = Paguri diahole). "Herbe des pintades. » La plante est consommée, en herbe seulement, par les chevalix. bœufs, moutons, chèvres (Garoua 594).

42. Eragrostis cambessediana Steud. - (Saraho walodyi.) Graminée des terres humides. Consommée en herbe et en grains, par les boeufs; moutons, chèvres. Non consommée par les chevaux (Garoua 597).

43. Eragrostis cilianensis Lutati. - (Lemno walowol [Garoua] $=$ Sihuko walowol [Maroua] $=$ Tappo walowol [Garoua]). Graminée des zones marécageuses. La paille est utilisée pour faire des liens. Elle sert à la couverture des cases, pour la confection de seccos fins destinés au couchage. La plante est consommée, en herbe et en grains, par les chevaux, bœufs, moutons, chèvres (Garoua 566).

44. Eragrostis ciliaris R. Br. - (Bitye dombi = Wudo kare). La plante est consommée, en herbe et en grains, par les chevaux, boufs, moutons, chèvres (Garoua 64, 504).

45. Eragrostis namaquensis Nees. - (Sinko Walowol $=$ Logoho walowol). Graminée des terrains marécageux. La paille est utilisée pour la confection de seccos fins pour le couchage, comme liens, pour la couverture des cases; la plante n'est consommée par le bétail qu'en cas de pénurie d'autres fourrages (Garoua 473).

46. Eragrostis pilosa Beauv. - (Hudo walowol [Garoua] $=$ Gabbel sarayel [Maroua] $=$ Buhirdi dabale [Garoua] $=$ Dombiho walowol). Graminée des terres humides, elle est consommée, en herbe et. en grains, par les chevaux, bœufs, moutons, chèvres (Garoua 216, 641).

47. Eragrostis squamata Steud. - (Hudo bodiel [Garoua $=$ Djalbatariho $[$ Maroua $]=$ Sammereho [Garoua] = Gawri tcholli [Garoua]). "Herbe des lapins »-« Mil des oiseaux ». La plante est pâturée, 
en herbe et en grains, par les chevaux, bceufs, moutons, chères (Garoua 215, 568).

48. Eragrostis tremula Hochst. - (Saraho [Garoua] = Sarawal yolde [Maroua]). Graminée des terres siliceuses, consommée, en herbe et en grains, par les chevaux, boufs, moutons, chèvres (Garoua 198).

49. Eragrostis turgida de Wild. - (Sehuko [Garoua] = Hudo walowol [Garoua] = Gawri tcholli) . Graminée des terres argileuses; la plante est consommée, en herbe et en grains, par les chevaux, bceufs, moutons, chères (Garoua 157).

50. Eragrostis non déterminé - (Sihuko walowol [Maroua] = Tappo walowol [Garoua] = Sarawal walowol). Graminée des terres humides. La paille est utilisée pour la confection de seccos fins pour le couchage, de liens, cordeaux. La plante est consommée, en herbe et en grains par les chevaux, bceufs, moutons, chères (Garoua 165).

51. Eragrostis non déterminé. - (Iludo walowol [Garoua] = Wytio direho walowol [Maroua] = Mubarawal bafere). Graminée des terres silico-argileuses, elle est consommée, en herbe seulement, par les chevaux, bœufs, moutons, chèvres (Garoua 476, 479).

52. Eriochloa acrotricha Hack ex Thell. - (Hudo yolde [Garoua] = Hudo tabedye [Garoua] = Gawri tcholli). Graminée des terres légères, humides, la plante est consommée, en herbe et en grains, par les chevaux, bœufs, moutons, chèvres (Garoua 354).

53. Hackelochloa granularis O. Ktze. - (Katkade bafedye $=$ Katakde gorde). Graminée pâturée, en herbe et en grains, par les chevaux, bœufs, moutons, chèvres (Garoua 67).

54. Heteropogon contortus Hoem. et Schult. (Tielbi bodedyi [Garoua] = Damsali gaduruho [Maroua] - Hettio soyboho [Maroua]). La paille est utilisée pour la couverture des cases. La plante est pâturée, en herbe et en grains par les bovins, ovins, caprins. Elle n'est consommée par les chevaux qu'en cas de pénurie d'autres fourrages (Garoua 63).

55. Hyparrhenia diplandra Stapf. - (Sehuko sadoyi $=$ bubelho). La paille est utilisée pour la couverture des casés. La plante est pâturée par les bovidés seulement (Garoua 163).

56. Hyparrhenia dissoluta Hubb. - (Djigaldji [Garoua] = Lilahadji [Garoua] = Sobarlaho mayo [Maroua]). Graminée poussant à proximité des cours d'eau. La paille est utilisée pour la couverture des habitations, pour la confection de seccos de clôture. La plante est consommée, en herbe seulement, par les chevaux, boeufs, moutons, chèvres (Garoua 174, 627).

57. Hyparrhenia rufa Stapf. - (Wedeho balwa hore $=$ wodeho walowol = Tielbiho [Garoua] $=$ Titidyï sadoyi [Maroua] = Sobarlaho [Maroua]). Graminée des terres argileuses, fraîches. La paille est utilisée pour la couverture des cases, pour la confection de seccos de clôture, de cordeaux. La plante est pâturée, en herbe seulement, par les bovins, ovins, caprins. Les chevaux ne la consomment pas (Garoua 5, 491, 515).

58. Hyparrhenia subplumosa Stapf. - (Tielbi [Garoua] = Sukahore [Maroua] = Mubarawal sukahore [Maroua]). La plante est consommée, en herbe seulement, par les chevaux, boufs, moutons, chèvres (Garoua 610).

59. Inperata cylindrica Beauv, - (Soho). Graminée envahissante des terres en cours de dégradation (Tranh des Indochinois). Les rhizomes traçants s'enfoncent à grande profondeur et sont difficiles à extirper. La plante, très siliceuse, n'est pas pâturée par le bétail. La paille est utilisée pour la couverture des cases, pour la confection de seccos fins (Secco soho), de sparterie : corbeilles, couvre-plats (Peketeho, Toleho), de cordeaux, de sacs grossiers pour les récoltes (Garoua 13).

60. Jardinea congoensis Franch. - (Hiware = Hudo kassar [Maroua]). Graminée des terrains marécageux, elle a une saveur amère et n'est pas consommée par le bétail. Elle serait toxique' pour les sauterelles. La paille est utilisée pour la confection de seccos de clôture, de seccos de couchage, de stores (Kassars), de corbeilles (Garoua 244).

61. Leersia hexandra Swartz. - (Label boraki [Garoua] = Dedoyel [Maroua]). Les feuilles, très râpeuses, ne sont pas consommées par'le bétail [Garoua 480].

62. Leptochloa coerulescens Steud. - (Jamuko, mayo [Garoua]). La plante est consommée, en herbe et en grains, par les chevaux, bœufs, moutons, chevres (Caroua 631).

63. Loudetia phragmitoides C. E. Hubb. - (Mayi dugudji [Garoua] = Hudo lesso [Garoua]). Graminée aquatique dont les tiges sont utilisées pour la confection de lits indigènes (Taras). La plante n'est pas consommée par le bétail (Garoua 478).

64. Loudetia simplex C. E. Hubb. - (Sersi [Garoua] $=$ Euhirdi sadowol [Maroua] = Tielbi bodedyi [Maroua]). La paille est utilisée pour la confection de seccos (cloture et couchage), de balais, de cordeaux, pour la couverture des cases. La plante est pâturée en herbe seulement, par les chevaux, boufs, moutons, chèvres (Garoua 518, 598).

65. Melinis minutiflora Beauv. - Graminée d'altitude. Elle existe en peuplements purs sur les plateaux dominant Poli. Plante excellente pour l'engraissement du betail. Les feuilles velues et visqueuses sécrètent une oléorésine répandant une forte odeur de réglisse. Cette graminée serait d'origine américaine. Elle permet la destruction des graminées rhizomateuses, telles que l'Imperata. 
66. Oryza barthii A. Chev, - Riz sauvage. (Nanare $[$ Garoua $]=$ Marori belli $[$ Maroua] $=$ Marori ladde $[$ Maroua] $=$ Nanare dabale [Garoua]), Les grains sont consommés en cas de disette alimentaire. La plante est pâturée, en herbe seulement, par les chevaux, bœufs, moutons, chèvres. L'envahissement des rizières cultivées par le Riz sauvage est un signe de l'appauvrissement du sol. Mais cet envahissement constitue un apport important d'humus (Garoua 247).

67. Oryza sativa Linn. - Riz cultivé (Marori). C'est la céréale alimentaire la plus importante du globe. Localement, les grains sont quelquefois consommés crus, le plus souvent bouillis ou après réduction en farine. L'eau de cuisson du riz (eau de riz) soigne les maux intestinaux.

68. Panicum anabaptistum Steud. - (Logoho [Garoua] = Sehuko [Maroua] = Buhurdi [Maroua]). La paille est utilisée pour la couverture des habitations, elle sert à la confection de seccos (clôture et couchage), de balais. La plante n'est pas consommée par le bétail (Garoua 285).

69. Panicum humile Nees. - (Sehisehideho). La plante est pâturée, en herbe et en grains, par les chevaux, boufs, moutons, chèvres (Garoua 637).

70. Panicum Iongijubatum Stapf. - (Hudo mayo [Garoua] $=$ Hudo belli [Garoua] $=$ Gawri tcholli [Maroua]). Graminée fréquente aux abords des cours d'eau. La plante est consommée, en herbe seulement, par les chevaux, boeufs, moutons, chèvres (Garoua 161, 186).

71. Panicum repens L. - (Hudo bedi = Bandeho). La paille est utilisée pour confectionner des liens, des cordeaux. La plante est consommée, en herbe et en grains, par les chevaux, bœufs, moutons, chèvres (Garoua 624).

72. Panicum non déterminé. - (Tappo bogol $=$ Tappo bafere - hirhillo). La paille est utilisée pour la confection de seccos fins pour le couchage, de cordeaux. La plante est consommée, en herbe et en grains, par les chevaux, boufs, moutons, chèvres (Garoua 486).

73. Panicum non déterminé. - (Badeho [Garoua] = Pagari yolde [Maroua]). Graminée des terres légères. La plante est consommée, en herbe et en grains, par les chevaux, bœufs, moutons, chèvres (Garoua 589).

74. Panicum non déterminé. - (Wytio dire [Garoua] = Pagari wolowol [Maroua]). Graminée des terres argileuses fraîches, elle est pâturée, en herbe et en grains, par les chevaux, boufs, moutons, chèvres (Garoua 609).

75. Panicum non déterminé. - (Maroriho walowol [Garoua] $=$ Hudo walowol [Maroua] = Paguri mayo [Maroua]). Graminée des terres marécageuses. La plante est consommée en herbe et en grains, par les chevaux, bœufs, moutons, chèvres (Garoua 290, 517).

76. Panicum non déterminé. - (Paguri walowol [Maroua] = Paguri diahole [Garoua] = Hudo patuyi [Garoua] = Buhirdi walowol [Garoua]). La paille sert à la confection de balais. La plante est pâturée, en herbe et en grains, par les chevaux, bœufs, moutons, chèvres (Garoua 167, 516).

77. Panicum non déterminé. - (Saraho djarendi mayo [Garoua]). Graminée des terrains sableux. La plante est consommée, en herbe et en grains, par les chevaux, bceufs, moutons, chèvres (Garoua 633).

78. Panicum non déterminé. - (Sarawal dunde mayo [Garoua]). La plante est pâturée, en herbe et en grains, par les chevaux, boeufs, moutons, chèvres (Garoua 636).

79. Paspalidium (?). - (Kayari). Les graines sont utilisées pour l'alimentation humaine, en cas de disette de graminées vivrières. Lu plante est pâturée, en herbe et en grains, par les chevaux, boufs, moutons, chèvres (Garoua 206, 482).

80. Paspalum scrobiculatum Linn, var. polystachyum Stapf. - (Kumbo betta [Maroua] = Bettaho [Maroua] = Bungo [Garoua]). Cette graminée n'est utilisée comme fourrage 'qu'en cas de pénurie d'aliments pour le betail. Elle est réputée comme donnant aux chevaux des maux de dents (Garoua 158).

81. Paspalum scrobiculatum. - (Kumbo betta $[$ Maroua] $=$ Bettaho [Maroua] = Bungo [Garoua]). Cette graminée et la précédente sont considérées localement comme une seule et même espèce (Garoua 616).

82. Paspalum non déterminé. - (Vetalde mayo [Garoua] = Bungel bodeyel [Maroua]). En herbe, la plante est consommée par les chevaux, bœufs, moutons, chèvres. En grains, elle est pâturée par les bœufs seulement (Garoua 634).

83. Pennisetum pedicellatum Trin. - (Bulude $=$ Wulude). La paille est utilisée pour la couverture des cases. La plante est consommée, en herbe et en grains, par les chevaux, bœufs, moutons, chèvres (Garoua 93).

84. Pennisetum purpureum Schum. - " Herbe à éléphants ». - (Pandir [Garoua] -- Tolore [Garoua] = Soko kore [Garoua] = Tyiseri [Sissongo en Douala]). La paille est utilisée pour la fabrication des "Taras ", pour la couverture des habitations. La plante est consommée, en herbe et en grains, par les bovins, ovins, caprins. Les chevaux la consomment en herbe seulement. Le " sissongo " produit beaucoup d'humus et maintient la fertilité du sol mais il est, par la suite, difficile à éliminer (Garoua 255). 
85. Pennisetum ramosum Sweilf. - (Wutalde [Maroua] = Wytio dire [Garoua]). La paille est utilisée pour la couverture des cases. La plante est pâturée, en herbe et en grains, par les chevaux, bœufs, moutons, chèvres (Garoua 514).

86. Pennisetum thyphoideum. - Mil chandelle ou Petit mil. Céréale vivrière. Il en existe deux variétés : Muri, variété précoce, d'une croissance de 4 mois; Yadiri, d'une croissance de 6 mois Les grains sont quelquefois consommés crus, le plus souvent cuits, après réduction en farine. Les grains pilés avec de l'ail et absorbés dans du lait, soignent les rhumatismes.

87. Pennisetum non détorminé. - (Wytio wandu walowol [Garoua] = Huluko walowol [Maroua]). Graminée des terrains marécageux. La paille est utilisée pour la còverture des habitations. La plante n'est pâturée, en herbe et en grains qu'en cas de pénurie d'autres fourrages (Garoua 596).

88. Perotis indica Beauv. - (Wytio domru = Bitye badi baledyi). La plante est pâturée, en herbe et en grains, par les chevaux, bocufs, moutons, chèvres (Garoua 501).

89. Phragmites communis Trin. - Roseau. (Golbi $=$ Golbi lesso). Graminée. aquatique. Les tiges sont utilisées pour la confection des lits indigènes (taras), des tuyaux de pipe, pour la couverture des cases (coussinets). La plante est consommée, en herbe seulement, par les bovins, ovins, caprins. Elle n'est pas consommée par les chevaux (Garoua 259).

90. Rhynchelytrum repens C. E. Hubb. - (Sabereho [Garoua] = Mbayeriho $[$ Maroua] $=$ Lebreho [Garoua] $=$ Lebreho tabedye $=$ Dioldioldu $=$ Tielbi golbol $=$ Hadiardial $\left[\mathrm{N}^{`}\right.$ Gaoundéré $]$. La plante est consommée, en herbe seulement, par les chevaux, bœufs, moutons, chevres (Garoua 172, 173, 499).

91. Rhytachne triaristata Stapf. - (Hudo marori [Garoua] = Hudo sadoyi [Maroua]. La plante est pâturée, en herbe et en grains, par les chevaux, boufs, moutons, chèvres (Garoua 485).

92. Rottboellia exaltata Linn. - (Niello). Considérée comme un bon fourrage, celle graminée est pâturée, en herbe et en grains, par les chevaux, bœufs, moutons, chèvres (Garoua 162, 492).

93. Saccharum officinarum Linn. - Canne à sucre. - (Yombe rake = lamarudu). Elle est cultivée dans le Nord Cameroun pour ses tiges sucrées, consommées crues ou cuites dans la bouillie. Par cuisson prolongée dans l'eau, on obtient un sirop utilisé comme boisson.

94. Saccharum spontaneum Linn. var. aegyptiacum Hack. - (Kahuledji.) Graminée spontanée des terrains humides, des abords'de cours d'edu.
Les tiges sont utilisées pour la confection de taras, de flèches, de coussinets pour la couverture des cases. La plante est consommée, en herbe seulement, par les bœufs, moutons, chèvres. Elle n'est pas consommée par les chevaux. (Garoua 178).

95. Sacciolepis interrupta Stapf. - (Burgu pabi). La plante est pâturée, en herbe et en grains, par les chevaux, boufs, moutons, chèvres (Garoua 488).

96. Sacciolepis micrococca Mez. - (Wytio domru). La plante est consommée en herbe et en grains, par les chevaux, bœufs, moutons, chèvres (Garoua 608).

97. Sacciolepis non déterminé - (Salteho sebore). La plante est consommée, en herbe et en grains par les chevaux, bocufs, moutons, chèvres (Caroua 638).

98. Schizachyrium exile Stapf. - (Mubarawal = Hudo bembe). La paille, hachée, est incorporée à l'argile utilisée pour les constructions en « potopoto ». La plante n'est pas consommée par le bétail (Garoua 505).

99. Schizachyrium semiberbe Nees. - (Wodeho titidyi [Garoua] = Hudo titidyi yolde [Garoua] = Titidyi bodeyi walowol [Maroua] = Wodeho ranhia hore [Maroua]). La paille est utilisée pour la confection de seccos de clôture, pour la couverture des cases. La plante est consommée, en herbe seulement, par les bovins, ovins, caprins. Elle n'est pas consommée par les chevaux (Garoua 164, 493, 607).

100. Setaria barbata Kunth. - (Paguri salodyi = Paguri diahole [Garoua] = Badeho [Maroua] $=$ Wedereho). La plante est pâturée en herbe et en grains, par les chevaux, bœufs, moutons, chèvres. Les graines sont recherchées par les francolins (Garoua 185).

101. Setaria pallidifusca Stapf. et Hubb. - (Bitye badi walodyi $=$ Bitye badi danedye $=$ Wanduho walowol). La plante est consommée en herbe et en grains par les chevaux, boufs, moutons, chevres (Garoua 159, 218, 487, 639).

102. Setaria sphacelata Stapf. et Hubb. - (Sorbereho mayo). Graminée fréquente aux abords des cours d'eau. La paille est utilisée pour la couverture des habitations, pour la confection de seccos de clôture. La plante est consommée, en herbe et en grains, par les chevaux, bœufs, moutons, chèvres (Garoua 623).

103. Setaria verticillata Beauv. - (Niakabre). Les graines sont munies de crochets et les épis s'accrochent facilement aux vêtements. La plante est consommée, en herbe et en grains, par les chevaux, boufs, moutons, chèvres (Garoua 209, 554).

104. Sporobolus aequiglumis Stapf. - (Hudo walowol [Garoua] = Hudo bodiel [Garoua] = Gasa debbo [Maroua] $=$ Hore pul debbo [Maroua], "Cheveux de feinne Peul »). La plante est pâturée. 
en herbe et en grains par les chevaux, boufs, moutons, chevres (Garoua 506).

105. Sporobolus festivus Hochst. - (Bodiel = Manga dangula = Hudo walowol). En herbe seulement, la plante est pâturée par les chevaux, bceufs, moutons, chères, lapins (Garoua 212).

106. Sporobolus pyramidalis P. B. - (Lemno). La paille est utilisée pour la confection de seccos fins pour le couchage, de cordeaux, de liens, de balais, pour la couverture des cases d'habitation. La plante est pâturée, en herbe et en grains, par les bovidés seulement (Garoua 184).

107. Sorghum arundinaceum Stapf. -(Gayori). Graminée spontanée dont la décoction de feuilles est utilisée pour teindre en rouge les calebasses, les nattes. En cas de disette, les grains sont utilisés pour I alimentation humaine. La plante n'est pas consommée par le bétail. A l'état jeune, elle est réputé comme toxique (Garoua 213).

108. Sorghum aterimum Stapf. - (Tingeri). Graminee spontanée. Les graines, sous forme de farine, entrent dans l'alimentation humaine en cas de disette de céréales vivrières cultivées. La plante. en herbe et en grains, est pâturée par les chevaux, bœufs, moutons, chèvres (Garoua 587).

109. Sorghum caudatum Stapf. - (Gardawal [Garoua] $=$ Yambe [Garoua] $=$ Gabadelwal [Maroua]). Graminée cultivée comme plante tinctoriale. La décoction des liges dome un colorant rouge utilisé pour les peaux, les nattes, les calebasses.

110. Sorghum vulgare Brot. - Sorgho ou Gros mil. Il existe de nombreuses variétés se répartissant en précoces (Djigari en Fulfudé), intermédiaires (Mbalyeri en Fulfudé), tardives (Mils de décrue), (Muskuari en Fulfuldé). Le mil est utilise pour l'alimcntation humainc, quelquefois cru, le plus souvent cuit, sous forme de farine. Il entre dans la nourriture des chevaux. Les populations païennes (Kirdis) en font une boisson fermentée (bière de mil) dont la consommation est très impor tante.

111. Tetrapogon spathaceus Mack. - (Hude bodiel $=$ Lebre bodiel = Damaliliel bodiel = Damaliliel Kare). La plante est pâturée, en herbe et en grains, par les chevaux, boufs, moutons; chères, lapins sauvages (Garoua 494).

112. Thelepogon elegans Roth. ex Roem. et Schult. - (Katkade = Katkade debbe $=$ Katkade yolde). Graminée des terrains siliceux, consommée en herbe et en grains, par les chevaux, boufs, moutons, chèvres. Chez le cheval, la plante a une action vermifuge (Garoua 304, 496).

113. Tristachya thollonii Franch. - (Tappo /Garoua] = Tapraneho [Maroua]). La paille est utilisée pour la confection de seccos, de liens, de cordeaux, pour la couverture des cases. La plante n'est pas pâturćc par le bćtail (Garoua 620).

114. Urochloa trichopus Stapf. - (Badeho yolde [Garoua] = Wabareho [Maroua]). La plante est pâturee, en herbe et en grains, par les chevaux, qœeufs, moutons, chèvres (Garoua 588).

115. Vetiveria nigritana Stapf. - (Sodornde = Sodorko). La paille est utilisée pour la fabrication de seccos de clôture, de vans, de cordeaux, de couvre-calebasses (sparterie), de nattes, de chapeaux, pour la couverture des cases. La racine « donne bon goût » à l'eau de boisson dans laquelle elle est mise à macérer. La racine, écrasée avec du natron, soigne les maux de dents. La piante est consommée, à l'état jeune seulement par les chevaux, bœufs, moutons, chèvres (Garoua 176).

116. Zea mays Linn. - Maïs. - (Butali [Garoua] = Masardyi [Marona]). Céréale de soudure, sa récolte précoce permet la liaison avec la récolte du mil «Djigari ». Il est consommé cluit sous forme de farine, mais, le plus souvent, quand il est récemment récolté, il est consommé après cuisson sous la cendre des épis entiers. Les Kirdis en font une boisson fermentée.

\section{SUMMARY}

Gramineae of the Northern Cameroons and their uses.

The writer reviews 116 gramineae of the region called "The Chad Plain" in the Northern part of the Cameroons, between Ngaoundere and Lake Chad. He indicates their names in two dialects of the "Fulfulde" language or "Peule" of the Northern Cameroons, and their various utilization as fodder, as medicinal herbs, as food for man, and as material for the construction of huts, etc.

\section{RESUMEN}

Gramineas del Camerún septentrional y su utilización.

El autor pasa revista a 116 gramineas de la región llamada " Ilanura del Tchad ", en la parte septentrional del Camerún, entre Ngaoundéré y el lago 'Tchad. Indica sus nombres en los dos dialectos de la lengua "Fulfulde " o peule del Camerún septentrional, y sus diversos empleos, como forrages, plantas medicinales, alimento para el hombre, material de construcción de chozas, etc. 